\title{
A Rare Case of Colonic Metastases From Tonsillar Carcinoma: Case Report and Review of Literature
}

\author{
Hassan Tariq ${ }^{\mathrm{a}}$, Muhammad Umar Kamal ${ }^{\mathrm{a}, \mathrm{c}}$, Shehriyar Mehershahi ${ }^{\mathrm{a}}$, Muhammad Saad ${ }^{\mathrm{a}}$, \\ Sara Azam ${ }^{a}$, Kishore Kumara ${ }^{\mathrm{a}}$, Masooma Niazib, \\ Jasbir Makkera, Myrta Daniel ${ }^{\mathrm{a}}$
}

\begin{abstract}
The incidence of tonsillar cancer has increased by four times in the United States over the last few decades likely due to recent increase in human papilloma virus (HPV) infections. The stage of the tumor predicts likelihood of metastasis, with advanced stages associated with higher chances of metastasis. The squamous cell carcinomas (SCCs) of the head and neck commonly metastasize to the lung, bone and liver in descending order. Tonsillar cancer rarely involves the small bowel and our review of the literature did not reveal any reported case of metastasis to the colon/large bowel. Our patient had locally metastatic tonsillar cancer, treated with partial pharyngectomy and selective neck dissection but later developed several bone and colonic metastases concurrently, likely secondary to hematogeneous dissemination after a few months of therapy. To the best of our knowledge, large bowel metastasis from head and neck SCC has never been reported in the literature. In these patients presenting with atypical gastrointestinal symptoms, a high index of suspicion should be maintained to determine the extent of metastasis and identify other metastatic sites.
\end{abstract}

Keywords: Gastrinoma; Colonic metastases; Tonsillar carcinoma; Head and neck cancer

\section{Introduction}

The incidence of tonsillar cancer, a subtype of the head and neck $(\mathrm{H}$ and $\mathrm{N})$ cancers has increased by four times in the United States over the last few decades [1]. The incidence rates differ worldwide based on the etiologies and region. A recent increase in human papilloma virus (HPV) infections and smok-

Manuscript submitted December 6, 2017, accepted January 3, 2018

aDepartment of Medicine, Bronx Lebanon Hospital Center, Bronx, NY 10457, USA

bDepartment of Pathology, Bronx Lebanon Hospital Center, Bronx, NY 10457, USA

${ }^{\mathrm{c} C o r r e s p o n d i n g ~ A u t h o r: ~ M u h a m m a d ~ U m a r ~ K a m a l, ~ D e p a r t m e n t ~ o f ~ M e d i c i n e, ~}$ Bronx Lebanon Hospital Center, 1650 Selwyn Ave., Suit \#10C, Bronx, NY 10457, USA. Email: Mkamal@bronxleb.org

doi: https://doi.org/10.14740/wjon1073w ing patterns has contributed to the upsurge of tonsillar cancer [1]. Stage of the tumor determines likelihood of metastasis, with advanced stages associated with approximately $15-20 \%$ chances of metastasis [2]. Squamous cell carcinomas (SCCs) of the $\mathrm{H}$ and $\mathrm{N}$ commonly metastasize to the lung, bone and liver in descending order [3]. Some case reports have mentioned metastasis to the small bowel [4]. Tonsillar carcinoma (TC) usually metastasizes locally to the cervical lymph nodes [5]. Early stage cancers rarely have distal metastasis [2] but advanced stage cancers can have metastasis beyond cervical lymph nodes. It spreads through the lymphatic and/or vascular channels [6].

\section{Case Report}

A 57-year-old man was evaluated in the emergency room (ER) of our hospital for complains of dizziness and near syncope while walking home earlier that day. He had started feeling generalized weakness and fatigue after he underwent neck surgery for a tonsillar lesion at another hospital 6 months earlier. He also reported unintentional weight loss of 20 pounds over 6 months along with loss of appetite. He denied any loss of consciousness, palpitation, shortness of breath, chest pain, hearing difficulty, headache, vision changes, nausea, vomiting, diaphoresis or fever. He had human immunodeficiency virus infection/acquired immunodeficiency syndrome (HIV/AIDS) with a CD4 count of less than 20. He was nonadherent to the anti-retroviral therapy. He also had chronic obstructive pulmonary disease and was using albuterol and steroid inhalers. His social history was significant for active cocaine abuse. He reported smoking one pack of cigarette per day for more than 35 years. Family history was significant for prostate cancer in father. His surgical records from the previous hospitalizations were obtained and revealed robotic resection of the left tonsil lesion and selective neck dissection 3 months prior with biopsy results showing left tonsillar SCC, P16+, stage T1N3M0. He did not receive any radiation or chemotherapy.

In the ER, his vitals were recorded as: pulse of 75 beats/ min, blood pressure 125/75 mm Hg with no orthostatic hypotension, respiratory rate of 16 breaths/min and oxygen saturation of $98 \%$ on room air. His physical exam was significant for severe cachexia with body mass index (BMI) of 19 . Conjunctival pallor was noted. His neck exam showed staples 


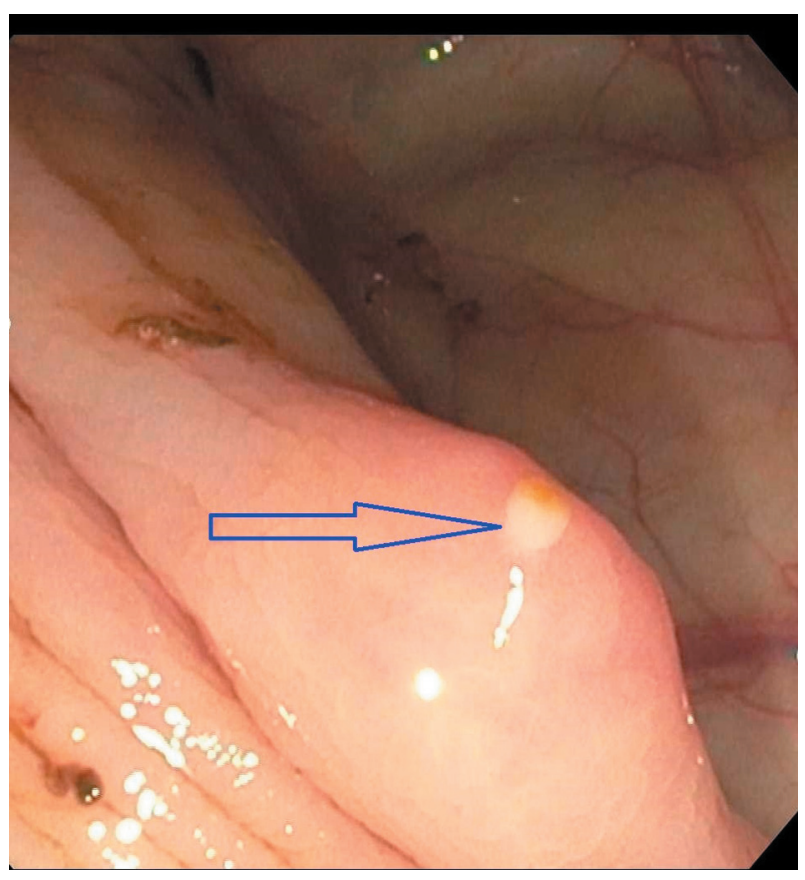

Figure 1. Cecal nodular erosion on colonoscopy (arrow).

on left side with a clean wound site. He was also noted to have left second toe swelling and tenderness with clammy extremities and adequate pulsations bilaterally. A computed tomography $(\mathrm{CT})$ scan of head was done to evaluate dizziness and near syncope that showed old ischemic changes. Initial electrocardiogram (ECG) showed non-specific ST/T wave changes consistent with previous ECG. On laboratory evaluation, he was found to have iron deficiency anemia with hemoglobin of $6.4 \mathrm{mg} / \mathrm{dL}$ and troponins of 0.165 secondary to demand ischemia. Pulmonary embolism was ruled out by a CT angiogram.

He was admitted to the medical floor where he was given one packed red blood cells (RBCs) transfusion. He underwent

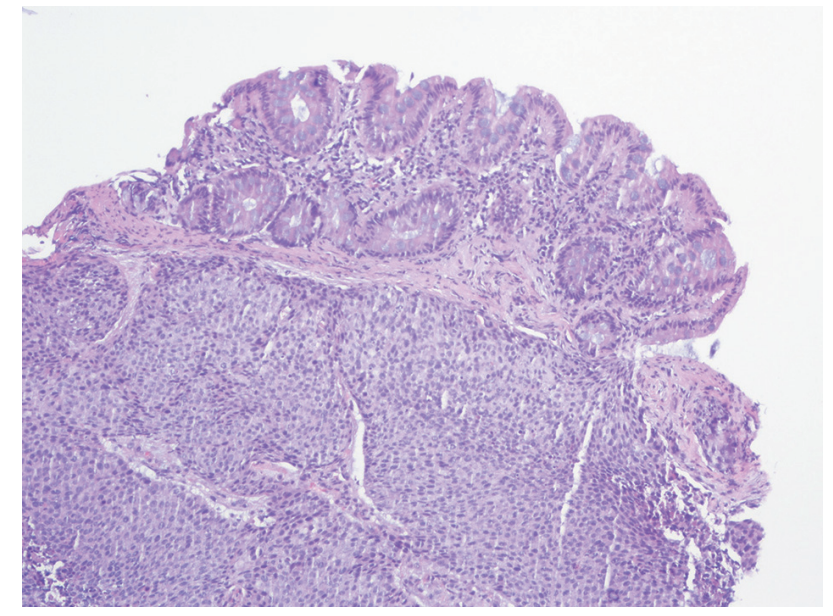

Figure 2. Poorly differentiated carcinoma metastatic to cecum. The micrograph shows colonic mucosa and submucosa which is diffusely infiltrated by metastatic carcinoma (H\&E, × 100 magnification).

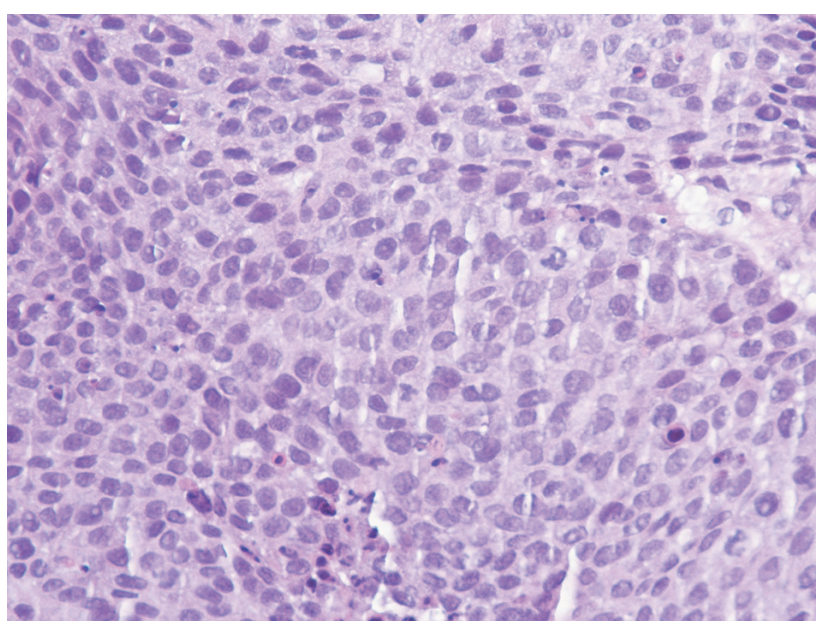

Figure 3. Poorly differentiated carcinoma metastatic to cecum showing sheets of malignant cells with nuclear pleomorphism and rare intracytoplasmic keratinization. No epithelial pearls or intercellular bridges or glandular pattern is seen (H\&E, magnification $\times 400)$.

an esophagogastroduodenoscopy (EGD) for evaluation of anemia that showed non-bleeding gastric ulcers that were clipped. CT scan of left foot showed acute osteomyelitis in second toe, so he was started on broad-spectrum antibiotics and bone biopsy was planned. After bone biopsy was performed, he had one episode of hematemesis with melena. He underwent a repeat upper endoscopy that showed no obvious source of bleeding. A colonoscopy was performed that showed $2-4 \mathrm{~mm}$ polyps in the colon with cecal nodular erosions (Fig. 1), which were biopsied. To our surprise, the colonic and bone biopsy results were reported as metastatic poorly differentiated carcinoma of tonsillar origin (Figs. 2 and 3). After discussion with patient and his family about plan of care and different treatment options, the patient wanted no surgical or oncological intervention and opted for palliative care.

\section{Discussion}

The distant metastasis of the $\mathrm{H}$ and $\mathrm{N}$ cancers after receiving radio/chemotherapy carries worse prognosis [7]. SCCs of the $\mathrm{H}$ and $\mathrm{N}$ commonly metastasize to the following organs in the descending sequence, i.e. lung $>$ bone $>$ liver [3]. Some cases of metastasis to the small bowel have been reported [4]. The review of the literature did not reveal any case of metastasis of tonsillar cancer to the colon/large bowel.

The overall incidence of distal metastasis of $\mathrm{H}$ and $\mathrm{N}$ cancer is $10-15 \%$ [8]. Our patient had locally metastatic disease treated with partial pharyngectomy and selective neck dissection but later developed several bone and colonic metastases noticed simultaneously during hospital admission. This is likely through hematogeneous dissemination after a few months of surgery.

To the best of our knowledge, large bowel metastasis from $\mathrm{H}$ and N SCC has never been reported in literature. Small bowel metastasis has been reported, and has been attributed to hematogenous dissemination or an abnormality of the lym- 
phovascular drainage [9]. Large bowel metastases can occur because of the transperitoneal seeding from adjacent organs like small intestine, ovarian, gastric and pancreatic tumors, but never reported from the of $\mathrm{H}$ and $\mathrm{N}$ cancers [10].

Multiple risk factors predict the risk of distant metastasis in $\mathrm{H}$ and $\mathrm{N}$ cancers. The most important factors reported in studies include neck lymph node involvement, site of primary tumor, stage and grade of tumor, and age of onset [11]. Our patient had excellent loco-regional control but presented with distant metastasis at uncommon site. It is imperative to follow similar cases to identify and manage distant metastasis in a timely manner. Chung et al reported the incidence and location of distant metastasis from the 475 patients with TC [12]. The tonsillar fossa was the most common region associated with distant metastasis and SCC was the most common type of tonsillar cancer. Extra-abdominal cancer metastasis to the colorectal region is rare. The primary cancers metastasizing to the colon include malignant melanoma, lobular breast carcinoma and lung cancer with estimated large bowel involvement of $27 \%, 5.3-12 \%$ and $2.2 \%$, respectively [13].

\section{Conclusions}

During review of the literature, no previous case reporting metastatic lesions of TC to the colon was found. In addition, metastasis to this region is not expected in a patient with TC and diagnosis is usually made based on histopathology analysis of the biopsied colonic tissue.

In patients presenting with atypical gastrointestinal symptoms, a high index of suspicion should be maintained to determine the extent of metastasis and identify other metastatic sites.

Local surgical resection of isolated colorectal (CR) metastasis may be beneficial in some patients but overall prognosis for non-primary CR cancers is dismal [7]. Therefore, palliative treatments should be offered in addition or as an alternative to adjuvant chemotherapy.

\section{Disclosures}

None.

\section{Author Contributions}

All authors have made contributions to the article and have reviewed it before submission.

\section{Consent}

Informed consent for participation was obtained from this patient.

\section{Conflict of Interest}

None of the authors have any financial conflicts of interest.

\section{References}

1. Frisch M, Hjalgrim H, Jaeger AB, Biggar RJ. Changing patterns of tonsillar squamous cell carcinoma in the United States. Cancer Causes Control. 2000;11(6):489-495.

2. Banerjee S, Kundu D, Mukherjee M, Maiti PK. Early stage squamous cell carcinoma of the tonsil presenting with multiple organ metastases including skin and brain after successful local treatment. Journal of Cancer Metastasis and Treatment. 2015;1(1):31.

3. Coca-Pelaz A, Rodrigo JP, Suarez C. Clinicopathologic analysis and predictive factors for distant metastases in patients with head and neck squamous cell carcinomas. Head Neck. 2012;34(6):771-775.

4. Dwivedi RC, Kazi R, Agrawal N, Chisholm E, St Rose $\mathrm{S}$, Elmiyeh B, Rennie C, et al. Comprehensive review of small bowel metastasis from head and neck squamous cell carcinoma. Oral Oncol. 2010;46(5):330-335.

5. Okura M, Aikawa T, Sawai NY, Iida S, Kogo M. Decision analysis and treatment threshold in a management for the N0 neck of the oral cavity carcinoma. Oral Oncol. 2009;45(10):908-911.

6. Irani S. Distant metastasis from oral cancer: A review and molecular biologic aspects. J Int Soc Prev Community Dent. 2016;6(4):265-271.

7. Hauswald H, Simon C, Hecht S, Debus J, Lindel K. Longterm outcome and patterns of failure in patients with advanced head and neck cancer. Radiat Oncol. 2011;6:70.

8. Leon X, Quer M, Orus C, del Prado Venegas M, Lopez M. Distant metastases in head and neck cancer patients who achieved loco-regional control. Head Neck. 2000;22(7):680-686.

9. Buyukcelik A, Ensari A, Sarioglu M, Isikdogan A, Icli F. Squamous cell carcinoma of the larynx metastasized to the ampulla of Vater. Report of a case. Tumori. 2003;89(2):199-201.

10. Ferlito A, Shaha AR, Silver CE, Rinaldo A, Mondin $\mathrm{V}$. Incidence and sites of distant metastases from head and neck cancer. ORL J Otorhinolaryngol Relat Spec. 2001;63(4):202-207.

11. Garavello W, Ciardo A, Spreafico R, Gaini RM. Risk factors for distant metastases in head and neck squamous cell carcinoma. Arch Otolaryngol Head Neck Surg. 2006;132(7):762-766.

12. Chung TS, Stefani S. Distant metastases of carcinoma of tonsillar region: a study of 475 patients. J Surg Oncol. 1980;14(1):5-9.

13. Lau CP, Hui EP, Chan AT. Complete small bowel obstruction caused by metastasis from primary nasopharyngeal carcinoma. Rare Tumors. 2009;1(1):e7. 